PROCEEDINGS OF THE

AMERICAN MATHEMATICAL SOCIETY

Volume 126, Number 12, December 1998, Pages 3589-3595

S 0002-9939(98)04433-5

\title{
LIPSCHITZ IMAGES WITH FRACTAL BOUNDARIES AND THEIR SMALL SURFACE WRAPPING
}

\author{
ZOLTÁN BUCZOLICH
}

(Communicated by J. Marshall Ash)

\begin{abstract}
Assume $E \subset H \subset \mathbf{R}^{m}$ and $\Phi: E \rightarrow \mathbf{R}^{m}$ is a Lipschitz $L$-mapping; $|H|$ and ||$H||$ denote the volume and the surface area of $H$. We verify that there exists a figure $F \supset \Phi(E)$ with $\|F\| \leq c_{L}|| H \|$, and, of course, $|F| \leq c_{L}|H|$, where $c_{L}$ depends only on the dimension and on $L$. We also give an example when $E=H \subset \mathbf{R}^{2}$ is a square and $\|\Phi(E)\|=\infty$; in fact, the boundary of $\Phi(E)$ can contain a fractal of Hausdorff dimension exceeding one.
\end{abstract}

\section{INTRODUCTION}

Related to a question of W. F. Pfeffer about bi-Lipschitz transformations of certain generalized integrals we had to deal with the following problem. Assume that the set $E \subset \mathbf{R}^{m}$ is a subset of a set $H \subset \mathbf{R}^{m}$ with $m$-dimensional measure $|H|$, and with surface area $\|H\|$, that is, the $m-1$-dimensional Hausdorff measure of the essential boundary of $H$ equals $\|H\|$. One can say that $E$ is wrapped into (the boundary of) $H$. The question which motivated this paper is the following: If $\Phi: E \rightarrow \mathbf{R}^{m}$ is a Lipschitz $L$-transformation, then is it true that one can wrap $\Phi(E)$ into a set which has "not much more" surface area and volume than that of $H$ ? To be more precise, is it true that there exists a constant $c_{L}$, depending only on the dimension and on the Lipschitz constant $L$, such that we can find a set $F$ containing $\Phi(E)$, and satisfying $\|F\| \leq c_{L}|| H \|$ and $|F| \leq c_{L}|H|$. In Theorem 1 we give an affirmative answer to this question. The first natural idea to prove Theorem 1 would be the usage of Kirszbraun's theorem [Fe, Th. 2.10.43.] to extend the Lipschitz $L$-mapping $\Phi$ onto $\mathbf{R}^{m}$ and use $F=\Phi(H)$. However this simple idea does not seem to work. In Theorem 2 we show that even in the simple case when $Q_{0} \subset \mathbf{R}^{2}$ is a square in the plane there exists a Lipschitz mapping $\Phi_{0}: \mathbf{R}^{2} \rightarrow \mathbf{R}^{2}$, such that the perimeter of $\Phi_{0}\left(Q_{0}\right)$ is of infinite measure. In fact, the boundary of $\Phi_{0}\left(Q_{0}\right)$ can contain a fractal, a self-similar set of Hausdorff dimension bigger than one.

The proof of Theorem 1 is not difficult. Considering a suitable covering of $H$ by open balls and the $\Phi$ image of this covering one can find the required figure. In our argument Besicovitch's covering theorem and the relative isoperimetric inequality

Received by the editors January 31, 1997 and, in revised form, April 21, 1997.

1991 Mathematics Subject Classification. Primary 28A75; Secondary 28A80, 26 B35.

Key words and phrases. Lipschitz mapping, surface, fractal.

This research was supported by the Hungarian National Foundation for Scientific Research, Grant Nos. T 019476 and T 016094. 
are used. We thank the referee for pointing out that the less known covering theorem [Fe, Corollary 4.5.4] could provide an even simpler argument; however to keep our paper self-contained we use the few lines longer alternate argument, which also contains the nice, and not widely known, key idea of [Fe, Corollary 4.5.4].

Interpreting BV sets (sets of bounded variation) as integral currents [Fe, Section 4.5.1] and applying the area theorem ([Fe, Theorem 3.2.3] and [Fe, Corollary 4.1.26]) it is easy to see that a one-to-one Lipschitz image of a BV set is again BV, that is, one-to-one Lipschitz images of sets of finite surface area are again of finite surface area. The mapping $\Phi$, constructed in the proof of Theorem 2 can be chosen to be conjugate (via a homeomorphism) to a folding of the square $Q_{0}$. Thus $\Phi$ is a two-to-one mapping at almost all points of $Q_{0}$, and it is one-to-one on the "folding" edge, which contains a fractal.

\section{PRELIMINARIES}

If $x, y$ are points of the Euclidean space $\mathbf{R}^{m}$, then the (Euclidean) distance of the points is denoted by $|x-y|$. The transformation $\Phi: \mathbf{R}^{m} \rightarrow \mathbf{R}^{m}$ is a Lipschitz $L$-mapping if

$$
|\Phi(x)-\Phi(y)| \leq L|x-y|
$$

holds for all $x, y \in \mathbf{R}^{m}$. The open ball centered at $x$ and of radius $r$ will be denoted by $B(x, r)$.

If $H$ is a subset of $\mathbf{R}^{m}$, then $|H|$ will denote its $m$-dimensional Lebesgue measure. (Note that for ease of notation the norm and the measure is denoted by the same symbol, though the domains of these symbols are different.) The $m$-1-dimensional Hausdorff measure of $H$ will be denoted by $\mathcal{H}(H)$.

A figure is a finite union of compact nondegenerate subintervals of $\mathbf{R}^{m}$.

Assume that $H \subset \mathbf{R}^{m}$ is measurable. The set of its Lebesgue density points is called the essential interior of $H$ and is denoted by int $H$, that is,

$$
\text { int }^{*} H=\left\{x \in \mathbf{R}^{m}: \lim _{\epsilon \rightarrow 0+} \frac{|H \cap B(x, \epsilon)|}{|B(x, \epsilon)|}=1\right\} .
$$

The set $\operatorname{cl}^{*} H=\mathbf{R}^{m} \backslash \operatorname{int}^{*}\left(\mathbf{R}^{m} \backslash H\right)$ is called the essential closure of $H$. Finally, $\partial^{*} H=\mathrm{cl}^{*} H \backslash$ int $^{*} H$ denotes the essential boundary of $H$. A set $H \subset \mathbf{R}^{m}$ is a $\mathrm{BV}$ set, a set of bounded variation, if it is bounded, measurable, and its perimeter $\|H\|=\mathcal{H}\left(\partial^{*} H\right)$ is finite. The family of BV sets arises quite often in geometric integration theory as the largest class where a reasonable surface integral can be defined, see, for example, [P, Section 2] where further references can be found as well. Clearly, figures are a very simple sort of BV sets.

Recall the following theorem from [M, Th. 2.7., p.30].

Besicovitch's covering theorem. There is a constant $c_{B}$ depending only on the dimension $m$ with the following property. Let $E$ be a bounded subset of $\mathbf{R}^{m}$, and let $\mathcal{B}$ be a family of balls such that each point of $E$ is the centre of some ball of $\mathcal{B}$. Then there are families $\mathcal{B}_{1}, \ldots, \mathcal{B}_{c_{B}}$ covering $E$ such that each $\mathcal{B}_{i}$ is disjoint, that is,

$$
E \subset \bigcup_{i} \bigcup \mathcal{B}_{i}
$$

and

$$
B \cap B^{\prime}=\emptyset \text { for } B, B^{\prime} \in \mathcal{B}_{i} \text { with } B \neq B^{\prime} .
$$


Besicovitch's covering theorem is stated in $[\mathrm{M}]$ for families of closed balls. Scrutinizing its proof one can easily see that the theorem holds for familes of open balls as well.

We will also use from [Z, Theorem 5.4.3., p.230] the following:

Relative isoperimetric inequality. If $H \subset \mathbf{R}^{m} a B V$-set, then for each ball $B(x, r) \subset \mathbf{R}^{m}$ we have

$$
\min \{|B(x, r) \cap H|,|B(x, r) \backslash H|\}^{\frac{m-1}{m}} \leq c_{\text {iso }} \mathcal{H}\left(B(x, r) \cap \partial^{*} H\right),
$$

where the constant $c_{i s o}$ depends only on the dimension.

Next we recall some properties of self-similar sets. For the details see, for example, [Fa, Ch.9., p.113-137]. Assume that the mappings $\varphi_{j}: \mathbf{R}^{m} \rightarrow \mathbf{R}^{m},(j=1, \ldots, k)$ are similarities with similarity ratios $c_{j}$. The (measurable) set $F$ is self-similar if

$$
F=\bigcup_{j=1}^{k} \varphi_{j}(F) .
$$

The mappings $\varphi_{j}$ satisfy the open set condition if there exists a non-empty bounded open set such that

$$
V \supset \bigcup_{j=1}^{k} \varphi_{j}(V),
$$

with the union disjoint. If the mappings $\varphi_{j}$ satisfy the open set condition, then the Hausdorff dimension of the self-similar set $F$, denoted by $s$, is given by

$$
\sum_{j=1}^{k} c_{j}^{s}=1 .
$$

In the special case when all $c_{j}$ 's have the same value, say $c$, then $s=\log (1 / k) / \log c$.

\section{MAIN RESULTS}

Theorem 1. Assume that $E \subset \mathbf{R}^{m}$ is compact, $\Phi: E \rightarrow \mathbf{R}^{m}$ is a Lipschitz Lmapping, and $H$ is a $B V$ set such that $E \subset$ int $^{*} H$. Then there exists a figure $F$ and a constant $c_{L}$, depending only on $L$ and on the dimension, such that $\Phi(E) \subset F$, $\|F\| \leq c_{L}|| H \|$, and $|F| \leq c_{L}|H|$.

Proof. For $x \in E$ put

$$
g_{x}(r)=\frac{|B(x, r) \cap H|}{|B(x, r)|} .
$$

Since $x \in \operatorname{int}^{*} H$ we have $\lim _{r \rightarrow 0+} g_{x}(r)=1$. On the other hand $H$ is bounded; hence $\lim _{r \rightarrow \infty} g_{x}(r)=0$. Since $g_{x}(r)$ is continuous, we can choose $r_{x}$, such that $g_{x}\left(r_{x}\right)=1 / 2$.

Using Besicovitch's covering theorem choose $c_{B}$ many classes $\left\{\mathcal{B}_{i}\right\}$, each consisting of balls $B\left(x, r_{x}\right)$, such that

$$
E \subset \bigcup_{i} \bigcup_{B\left(x, r_{x}\right) \in \mathcal{B}_{i}} B\left(x, r_{x}\right)
$$

and balls belonging to the same class $\mathcal{B}_{i}$ are disjoint. Since $E$ is compact we can also assume that each $\mathcal{B}_{i}$ consists of finitely many balls. 
Using the relative isoperimetric inequality and $g_{x}\left(r_{x}\right)=1 / 2$, we have

$$
\left(\left|B\left(x, r_{x}\right)\right| / 2\right)^{\frac{m-1}{m}} \leq c_{i s o} \mathcal{H}\left(B\left(x, r_{x}\right) \cap \partial^{*} H\right)
$$

for each $B\left(x, r_{x}\right)$, that is, there exists a constant $c_{i s o}^{\prime}$ such that

$$
\left\|B\left(x, r_{x}\right)\right\| \leq c_{i s o}^{\prime} \mathcal{H}\left(B\left(x, r_{x}\right) \cap \partial^{*} H\right) .
$$

Assuming that balls belonging to a class $\mathcal{B}_{i}$ are disjoint, we have

$$
\sum_{B\left(x, r_{x}\right) \in \mathcal{B}_{i}}\left\|B\left(x, r_{x}\right)\right\| \leq c_{i s o}^{\prime} \sum_{B\left(x, r_{x}\right) \in \mathcal{B}_{i}} \mathcal{H}\left(B\left(x, r_{x}\right) \cap \partial^{*} H\right) \leq c_{i s o}^{\prime}\|H\|,
$$

and

$$
\sum_{B\left(x, r_{x}\right) \in \mathcal{B}_{i}}\left|B\left(x, r_{x}\right)\right|=2 \sum_{B\left(x, r_{x}\right) \in \mathcal{B}_{i}}\left|B\left(x, r_{x}\right) \cap H\right| \leq 2|H|,
$$

for each $i \leq c_{B}$. Clearly, $\Phi\left(B\left(x, r_{x}\right)\right) \subset B\left(\Phi(x), L r_{x}\right) \subset Q_{\Phi(x)}$, where $Q_{\Phi(x)}$ is a closed cube centered at $\Phi(x)$ and of side length $2 L r_{x}$. Then there exists a constant $c_{Q}$, depending only on the dimension and on $L$, such that $\left\|Q_{\Phi(x)}\right\| \leq c_{Q}\left\|B\left(x, r_{x}\right)\right\|$, and $\left|Q_{\Phi(x)}\right| \leq c_{Q}\left|B\left(x, r_{x}\right)\right|$.

Let

$$
F=\bigcup_{i} \bigcup_{B\left(x, r_{x}\right) \subset \mathcal{B}_{i}} Q_{\Phi(x)}
$$

Then

$$
\begin{gathered}
\|F\| \leq \sum_{i} \sum_{B\left(x, r_{x}\right) \subset \mathcal{B}_{i}}\left\|Q_{\Phi(x)}\right\| \leq c_{B} c_{Q} c_{i s o}^{\prime}\|H\|, \\
|F| \leq \sum_{i} \sum_{B\left(x, r_{x}\right) \subset \mathcal{B}_{i}}\left|Q_{\Phi(x)}\right| \leq 2 c_{B} c_{Q}|H|,
\end{gathered}
$$

and clearly $\Phi(E) \subset F$. Therefore, one can define $c_{L}$ as the maximum of the constants $c_{B} c_{Q} c_{i s o}^{\prime}$ and $2 c_{B} c_{Q}$. This completes the proof.

Theorem 2. There exists a Lipschitz transformation $\Phi_{0}: \mathbf{R}^{2} \rightarrow \mathbf{R}^{2}$ and a square $Q_{0} \subset \mathbf{R}^{2}$ such that the Hausdorff dimension of $\partial^{*}\left(\Phi_{0}\left(Q_{0}\right)\right)$ is bigger than one; hence it is of non- $\sigma$-finite linear measure. Furthermore, $\Phi_{0}$ is two-to-one at almost all points of $Q_{0}$ and is one-to-one on the rest of $Q_{0}$.

Proof. It is enough to define a suitable Lipschitz transformation $\Phi$ on a rectangle $Q_{1}$ with vertices $(0,3),(48,3),(48,-3)$, and $(0,-3)$, since then, by using a suitable affine transformation, we can define $\Phi_{0}$ on $Q_{0}$, and using Kirszbraun's theorem we can extend $\Phi_{0}$ onto $\mathbf{R}^{2}$.

We construct $\Phi$ by a limit of a self-similar like repetition of the same mapping.

First we define a Lipschitz folding $\Phi_{1}$ of $Q_{1}$. Then we define 48 subrectangles $Q_{2}^{1}, \ldots, Q_{2}^{48}$ in $Q_{1}$, such that each $Q_{2}^{j}$ is similar to $Q_{1}$. Our mapping $\Phi$ will coincide with $\Phi_{1}$ on $Q_{1} \backslash \bigcup_{j=1}^{48} Q_{2}^{j}$. On the rectangles $Q_{2}^{j}$ we will do a folding process similar to the one in $Q_{1}$.

Put $s_{1}=(0,3), s_{2}=(1,3), s_{3}=(2,3), s_{4}=(2,-3), s_{5}=(1,-3)$, and $s_{6}=$ $(0,-3)$.

First we define $\Phi_{1}$ on the rectangle $S_{1}=s_{1} s_{2} s_{5} s_{6}$.

Choose the points $p_{1}, p_{2}, p_{3}$, and $p_{4}$ on the line $y=x$ such that $p_{1}=(0,0), p_{2}=$ $(0.1,0.1), p_{3}=(0.9,0.9)$, and $p_{4}=(1,1)$. Next we choose the points $q_{1}, q_{2}, q_{3}, q_{4}$, 
and $r_{1}, r_{2}, r_{3}, r_{4}$ on the lines $y=x-0.1$, and $y=x+0.1$, respectively, such that $q_{1}=(0,-0.1), r_{1}=(0,0.1), q_{2}=(0.15,0.05), r_{2}=(0.05,0.15), q_{3}=(0.95,0.85)$, $r_{3}=(0.85,0.95), q_{4}=(1,0.9)$, and $r_{4}=(1,1.1)$.

Denote by $Q_{2}^{1}$ the rectangle $q_{2} q_{3} r_{3} r_{2}$. Observe that $Q_{2}^{1}$ is similar to $Q_{1}$.

Let $\Phi_{1}$ equal the identity on the segments $p_{1} s_{6}$ and $s_{6} s_{5}$, and equal the reflection about the $x$-axis on the segments $p_{1} s_{1}$ and $s_{1} s_{2}$. On $r_{2} p_{2} p_{3} r_{3}$, that is, on the upper half of $Q_{2}^{1}$ we define $\Phi_{1}$ to be equal to the reflection about the $y=x$ line; on the lower half of $Q_{2}^{1}$, that is, on $p_{2} q_{2} q_{3} p_{3}$ we define $\Phi_{1}$ to be equal to the identity, that is, $\Phi_{1}$ is a lengthwise folding on $Q_{2}^{1}$.

At points of $S_{1}$, where we have not already defined $\Phi_{1}$, we extend its definition to meet the following requirements:

i) $\Phi_{1}$ is Lipschitz on $S_{1}$;

ii) $\Phi_{1}$ maps $S_{1}$ onto the trapezoid $p_{1} s_{6} s_{5} p_{4}$, which will be denoted by $T_{1}$;

iii) $\Phi_{1}$ is one-to-one on the segment $p_{1} p_{4}$ and is two-to-one at any other point of $S_{1}$; hence $\Phi_{1}\left(S_{1} \backslash Q_{2}^{1}\right)=T_{1} \backslash Q_{2}^{1}$

iv) $\Phi_{1}$ maps the line segment $s_{2} p_{4}$ onto the segment $p_{4} s_{5}$;

v) $\Phi_{1}$ equals the identity on the segments $p_{4} s_{5}, p_{1} p_{4}$.

It is an easy exercise, left to the reader, to show that the above conditions can be satisfied.

Next we define $\Phi_{1}$ on the strip $S_{2}=s_{2} s_{3} s_{4} s_{5}$. Denote by $\tau$ the reflection about the line $x=1$. For $p \in S_{2}$ set $\Phi_{1}(p)=\tau\left(\Phi_{1}(\tau(p))\right)$. Put $Q_{2}^{2}=\tau\left(Q_{2}^{1}\right)$. Then $Q_{2}^{2}$ is a rectangle similar to $Q_{1}$, and $\Phi_{1}$ on $Q_{2}^{2}$ equals a lengthwise folding of $Q_{2}^{2}$. Furthermore letting $T_{2}=\tau\left(T_{1}\right)$ we have the following properties satisfied.

i) $\Phi_{1}$ is Lipschitz on $S_{1} \cup S_{2}$;

ii) $\Phi_{1}$ maps $S_{1} \cup S_{2}$ onto $T_{1} \cup T_{2}$;

iii) $\Phi_{1}$ is one-to-one on the segments $p_{1} p_{4}$ and $p_{4} \tau\left(p_{1}\right)$, and is two-to-one at any other point of $S_{1} \cup S_{2}$; hence $\Phi_{1}\left(\left(S_{1} \cup S_{2}\right) \backslash\left(Q_{2}^{1} \cup Q_{2}^{2}\right)\right)=\left(T_{1} \cup T_{2}\right) \backslash\left(Q_{2}^{1} \cup Q_{2}^{2}\right)$;

iv) $\Phi_{1}$ equals the identity on the segments $p_{1} s_{6}, s_{6} s_{4}, s_{4} \tau\left(p_{1}\right)$ and $\Phi_{1}$ equals the reflection about the $x$-axis on the segments $p_{1} s_{1}, s_{1} s_{3}$, and $s_{3} \tau\left(p_{1}\right)$, that is, on the boundary of $S_{1} \cup S_{2}$ the mapping $\Phi_{1}$ coincides with a folding about the $x$-axis.

Denote by $\sigma_{j}$ the translation by the vector $(0,2 j)$. The strip bounded by the points $(j-1,3),(j, 3),(j,-3)$, and $(j-1,-3)$ is denoted by $S_{j}$. If $p \in S_{2 j-1} \cup S_{2 j}$, $(j=2,3, \ldots, 24)$, then put $\Phi_{1}(p)=\sigma_{j-1}\left(\Phi_{1}\left(\sigma_{j-1}^{-1}(p)\right)\right), Q_{2}^{2 j-1}=\sigma_{j-1}\left(Q_{2}^{1}\right)$, and $Q_{2}^{2 j}=\sigma_{j-1}\left(Q_{2}^{2}\right)$. Then one can easily see that $\Phi_{1}$ satisfies the following properties:

i) $\Phi_{1}$ is Lipschitz on $Q_{1}$, denote its Lipschitz constant by $L$;

ii) $\Phi_{1}$ coincides with a lengthwise folding on the boundary of $Q_{1}$, and on the entire closed rectangles $Q_{2}^{j},(j=1, \ldots, 48)$;

iii) If $g(x)$ denotes the sawtooth function which is periodic by 2, equals $y=x$ on $[0,1]$, and $y=-x+2$ on $[1,2]$, then $\Phi_{1}\left(Q_{1}\right)$ is the region bounded by the graph of $g$ and by the lower half of the boundary of $Q_{1}$;

iv) $\Phi_{1}$ is one-to-one on the part of the boundary of $\Phi_{1}\left(Q_{1}\right)$ which is on the graph of $g$; at any other point of $Q_{1}$ it is two-to-one.

Denote by $\varphi_{j}$ the similarities which map $Q_{1}$ onto $Q_{2}^{j},(j=1, \ldots, 48)$, and the lower half of $Q_{1}$ is mapped onto the lower half of $Q_{2}^{j}$. Observe that the contraction ratio of $\varphi_{j}$ equals $\sqrt{2} / 60$. 
For $p \in Q_{2}^{j}$ put $\Phi_{2}^{j}(p)=\varphi_{j}\left(\Phi_{1}\left(\varphi_{j}^{-1}(p)\right)\right)$. Then $\Phi_{2}^{j}$ is a Lipschitz $L$-mapping, and it coincides with $\Phi_{1}$ on the boundary of $Q_{2}^{j}$, since on this boundary they both equal a lengthwise folding of $Q_{2}^{j}$.

Put $\tilde{\Phi}_{1}(p)=\Phi_{1}(p)$ if $p \in Q_{1} \backslash \bigcup_{j=1}^{48} Q_{2}^{j}$, and $\tilde{\Phi}_{1}(p)=\Phi_{2}^{j}(p)$ if $p \in Q_{2}^{j}$.

Then $\tilde{\Phi}_{1}$ is a Lipschitz $L$-mapping. Indeed, assume $p, q \in Q_{1}$. If $p, q \notin \bigcup_{j=1}^{48} Q_{2}^{j}$, or there exists $j$ such that $p, q \in Q_{2}^{j}$, then $\left|\tilde{\Phi}_{1}(p)-\tilde{\Phi}_{1}(q)\right| \leq L|p-q|$ follows from the same property for $\Phi_{1}$, or for $\Phi_{2}^{j}$.

If $p \in Q_{1}$, and $q \in Q_{2}^{j}$, then the segment $p q$ intersects the boundary of $Q_{2}^{j}$. Denote this intersection point by $q^{*}$. Then

$$
\begin{aligned}
& \left|\tilde{\Phi}_{1}(p)-\tilde{\Phi}_{1}(q)\right| \leq\left|\tilde{\Phi}_{1}(p)-\tilde{\Phi}_{1}\left(q^{*}\right)\right|+\left|\tilde{\Phi}_{1}\left(q^{*}\right)-\tilde{\Phi}_{1}(q)\right| \\
& \quad=\left|\Phi_{1}(p)-\Phi_{1}\left(q^{*}\right)\right|+\left|\Phi_{2}^{j}\left(q^{*}\right)-\Phi_{2}^{j}(q)\right| \leq L\left(\left|p-q^{*}\right|+\left|q^{*}-q\right|\right)=L|p-q| .
\end{aligned}
$$

Finally if $p \in Q_{2}^{i}$ and $q \in Q_{2}^{j}$, then denoting by $p^{*}$ and $q^{*}$ the intersection points of $p q$ and the boundaries of $Q_{2}^{i}$ and $Q_{2}^{j}$, respectively, an argument, similar to the one in the above paragraph, can establish the Lipschitz $L$-property of $\tilde{\Phi}_{1}$.

It is also easy to see that $\tilde{\Phi}_{1}$ is two-to-one at almost all points of $Q_{1}$, the exceptional points are on the "folding edges" and at these points $\tilde{\Phi}_{1}$ is one-to-one.

Assume $k \geq 2, j_{1}, \ldots, j_{k} \in\{1, \ldots, 48\}$, and $p \in Q_{1}$. Put $\varphi_{j_{1}, \ldots, j_{k}}(p)=\varphi_{j_{1}} \circ \ldots$ $\circ \varphi_{j_{k}}(p), Q_{k+1}^{j_{1}, \ldots, j_{k}}=\varphi_{j_{1}, \ldots, j_{k}}\left(Q_{1}\right)$, and for $p \in Q_{k+1}^{j_{1}, \ldots, j_{k}}$, let $\Phi_{k+1}^{j_{1}, \ldots, j_{k}}(p)=$ $\varphi_{j_{1}, \ldots, j_{k}}\left(\Phi_{1}\left(\varphi_{j_{1}, \ldots, j_{k}}^{-1}(p)\right)\right)$. Denote $Q_{k+1}^{\prime}=\bigcup_{j_{1}, \ldots, j_{k} \in\{1, \ldots, 48\}} Q_{k+1}^{j_{1}, \ldots, j_{k}}$.

If $\tilde{\Phi}_{k-1},(k=2, \ldots)$ is given, then put $\tilde{\Phi}_{k}(p)=\tilde{\Phi}_{k-1}(p)$, for $p \in Q_{1} \backslash Q_{k+1}^{\prime}$, and $\tilde{\Phi}_{k}(p)=\Phi_{k+1}^{j_{1}, \ldots, j_{k}}(p)$, for $p \in Q_{k+1}^{j_{1}, \ldots, j_{k}}$. Then, it is not difficult to see, that $\tilde{\Phi}_{k}$ is a Lipschitz $L$-mapping and is a lengthwise folding on the boundaries of $Q_{k+1}^{j_{1}, \ldots, j_{k}}$ and on the sets $Q_{k+2}^{j_{1}, \ldots, j_{k+1}}=\varphi_{j_{1}, \ldots, j_{k}, j_{k+1}}\left(Q_{1}\right)$.

Since $\operatorname{diam}\left(Q_{k+1}^{j_{1}, \ldots, j_{k}}\right) \rightarrow 0$ as $k \rightarrow \infty$ it is easy to see that the sequence $\tilde{\Phi}_{k}$ uniformly converges on $Q_{1}$ to a Lipschitz $L$-mapping which we will denote by $\Phi$.

Put $Q_{\infty}=\bigcap_{k=1}^{\infty} Q_{k+1}^{\prime}$. Since $\Phi\left(Q_{1}\right)$ contains a little more than half of each $Q_{k+1}^{j_{1}, \ldots, j_{k}}$, examining our self-similar construction and using the definition of $\Phi$ on $Q_{k+1}^{j_{1}, \ldots, j_{k}}$, one can easily see that points of $Q_{\infty}$ belong to $\partial^{*}\left(\Phi\left(Q_{1}\right)\right)$.

Since $Q_{\infty}=\bigcup_{j=1}^{48} \varphi_{j}\left(Q_{\infty}\right)$, taking an open set $V$, which is slightly larger than $Q_{1}$, the open set condition also holds. Using that the $\varphi_{j}$ 's have contraction ratio $\sqrt{2} / 60$ we infer that

$$
\operatorname{dim}_{H}\left(\partial^{*}\left(\Phi\left(Q_{1}\right)\right)\right) \geq \operatorname{dim}_{H}\left(Q_{\infty}\right)=\frac{\log \left(\frac{1}{48}\right)}{\log \left(\frac{\sqrt{2}}{60}\right)}>1.03 .
$$

Finally, it is not difficult to see that $\Phi$ is two-to-one on $Q_{1}$ everywhere but the points of the "folding edge", where it is one-to-one. This completes our proof.

Remark. By modifying some constants in our construction and by using steeper sawtooth functions (for the internal folding edge in the definition of $\Phi_{1}$ ) one can get the Hausdorff dimension of $\partial^{*}\left(\Phi\left(Q_{1}\right)\right)$ arbitrarily close to 2 .

\section{REFERENCES}

[M] P. Mattila, Geometry of sets and measures in Euclidean spaces, Cambridge University Press, 1995. MR 96h:28006

[Fa] K. Falconer, Fractal Geometry, John Wiley \& Sons, 1990. MR 92j:28008 
[Fe] H. Federer, Geometric Measure Theory, Springer-Verlag, New York, 1969. MR 41:1976

[P] W. F. Pfeffer, The Gauss-Green theorem, Adv. Math. 87 (1991), 93-147. MR 92b:26024

[Z] W. P. Ziemer, Weakly differentiable functions, Springer-Verlag, New York, 1989. MR 91e: 46026

Eötvös Loránd University, Department of Analysis, Budapest, Múzeum krt 6-8, H-1088, Hungary

E-mail address: buczo@cs.elte.hu 